\title{
溶瘤新城疫病毒: 肿瘤免疫治疗时代的新机遇
}

\author{
杜莎 ${ }^{1 \dagger}$, 姜珂 ${ }^{2 \dagger}, 丁 \mathrm{c}^{3^{3}}{ }^{*}$, 许青 ${ }^{4^{*}}$, 李文斌 $5^{*}$, 孟松树 ${ }^{*}$
}

1. 大连医科大学肿瘤干细胞研究院, 大连 116044;

2. 上海交通大学医学院单细胞组学与疾病研究中心, 上海 200025 ;

3. 中国农业科学院上海兽医研究所禽类感染性疾病部, 上海 200241;

4. 同济大学附属上海市第十人民医院医学肿瘤部, 上海 200072 ;

5. 首都医科大学附属北京天坛医院神经外科中心神经肿瘤部, 北京 100070

$\dagger$ 同等贡献

* 联系人, E-mail: shoveldeen@shvri.ac.cn; xuqingmd@aliyun.com; liwenbin@ccmu.edu.cn; ssmeng@dmu.edu.cn

收稿日期: 2021-10-25; 接受日期: 2021-11-16; 网络版发表日期: 2021-12-15

摘要新型溶瘤病毒疗法治疗肿瘤, 是目前肿瘤免疫治疗研究的热点之一。溶瘤新城疫病毒(Newcastle disease virus, NDV)作为一种新型抗癌制剂, 在肿瘤治疗中利用肿瘤细胞中有缺陷的抗病毒防御, 选择性地感染复制和裂 解肿瘤细胞，增强肿瘤抗原暴露，促进免疫细胞的招募，激活抗肿瘤免疫应答，从而发挥抗肿瘤作用。基于溶瘤 NDV独特的抗肿瘤特性及其与患者免疫系统的相互作用，溶瘤NDV与其他免疫疗法联用可以增强抗肿瘤功效， 具有广阔的应用前景。本文从溶瘤NDV的结构、溶瘤机制、溶瘤NDV在肿瘤治疗领域的临床前研究和临床应 用、联合免疫治疗的应用及未来展望等方面进行综述.

关键词溶瘤病毒, 新城疫病毒, 肿瘤免疫治疗, 联合免疫治疗

癌症严重威胁人类健康，目前手术、放化疗仍是 癌症的主要治疗方法, 但这些传统治疗方法副作用大, 缺乏靶向性, 治疗效果欠佳. 溶瘤病毒(oncolytic virus, OV)是天然的或经人工改造的、能选择性地在肿瘤组 织中复制、直接或间接抑制肿瘤细胞生长的一类病 毒. 溶瘤病毒的溶解潜力与病毒种类、剂量、自然或 诱导的病毒趋向性、肿瘤细胞不同形式细胞死亡敏感 性等相关. 与其他治疗手段相比, 溶瘤病毒具备多个优 点，包括杀伤作用强、不易产生耐药性、副作用低、 低成本等. 此外, 多项临床实验显示, 除单独使用外, 溶瘤病毒与传统的癌症治疗相结合，显示出更好的疗
效. 目前，包括新城疫病毒(Newcastle disease virus, NDV)、腺病毒、腮腺炎病毒、单纯疮疹病毒、痘苗 病毒、呼肠弧病毒、甲型流感病毒、细小病毒等在内 的多种溶瘤病毒已进入临床试验阶段. 2000 2020年, 总计有97项相互独立的临床试验汇报溶瘤病毒治疗癌 症的临床试验结果，治疗的癌症类型包括黑色素瘤、 胃肠道肿瘤、胰腺癌等. 由国家管理机构批准的第一 个溶瘤病毒是ECHO-7肠道病毒RIGVIR，2004年在拉 脱维亚批准用于治疗皮肤黑色素瘤, 并在格鲁吉亚 (2015年)和亚美尼亚(2016年)获批. 2005年，中国食品 药品监督管理局(China Food and Drug Administration,

引用格式: 杜莎, 姜珂, 丁铲, 等. 溶瘤新城疫病毒: 肿瘤免疫治疗时代的新机遇. 中国科学: 生命科学, 2021, 51: 1623-1636 Du S, Jiang K, Ding C, et al. Oncolytic Newcastle disease virus: a new opportunity in the era of tumor immunotherapy (in Chinese). Sci Sin Vitae, 2021, 51: 1623-1636, doi: 10.1360/SSV-2021-0434 
CFDA)批准了上海三维生物公司开发的重组人 5 型腺 病毒注射液Oncorine(H101), 用于治疗头颈部肿瘤. 2015年, 美国食品药品监督管理局(U.S. Food and Drug Administration, FDA)和欧洲药品管理局(European Medicines Agency, EMA)先后批准了安进(Amgen)公 司开发的基于 1 型单纯疮疹病毒的IMLYGIC(talimogene laherparepvec, T-VEC), 用于治疗晚期黑色素瘤. 2021年, 第一三共(Daiichi Sankyo)公司宣布, 溶瘤病毒 Delytact(teserpaturev/G47D) 获得日本厚生劳动省的条 件性限时批准, 用于治疗恶性胶质瘤, 这是世界上首款 获批治疗原发性脑瘤的溶瘤病毒制剂. 2020年8月7日， 中国国家药品监督管理局药品评审中心发布了“关于 公开征求《溶瘤病毒类药物临床试验设计指导原则 (征求意见稿)》意见的通知”. 2021年，上海第十人民 医院许青教授组织撰写了溶瘤病毒专家共识第一版, 这是国内首次对溶瘤病毒研发提出的纲领性指导. 此 外，国外涌现出不少溶瘤病毒创新公司，如2001年纳 斯达克上市的Oncolytics Biotech，2018年纳斯达克上 市的Replimune, 在奥斯陆证券交易所OSE上市的溶瘤 病毒公司Targovax，在日本上市的溶瘤病毒公司Oncolys Biopharma，预计这些公司的溶瘤病毒产品将在 未来几年内获得批准. 国内目前也有不少创新企业以 及一些大型药企布局溶瘤病毒研发. 近期报道了乐普 生物购买了 GC Oncology公司开发的一款溶瘤病毒 CG0070(一种重组腺病毒Ad5)在中国区的开发、制造 及商业化的授权, 并已获准开展针对浸润性膀胱癌的 临床试验. 此外, 针对CG0070, 乐普生物将进一步开 发结直肠癌、肝癌及乳腺癌等其他适应症领域. 据不 完全统计，国内外大约有180多款溶瘤病毒项目处 于III期临床阶段, 覆盖 $80 \%$ 以上的实体瘤. 恶性胶质瘤 缺乏有效治疗手段，患者平均生存期仅为14 21个月. 由于血脑屏障的存在，很难开发出可以突破血脑屏障 的药物. 近期北京天坛医院李文斌教授启动一项针对 复发的中枢神经系统肿瘤的一款溶瘤病毒治疗临床试 验. 这项临床试验及日本批准的治疗胶质瘤的溶瘤病 毒试验表明，溶瘤病毒对一些死亡率高，缺乏有效治 疗手段的恶性肿瘤，如恶性脑胶质瘤，展示出独特的 临床效果.

近年来，免疫疗法的成功正在颠覆着整个肿瘤治 疗领域，该疗法包括免疫细胞疗法 ${ }^{[1]}$ 、肿瘤疫苗 ${ }^{[2]}$ 、 免疫检查点抑制 ${ }^{[3]}$ 等，其中最具代表性的是包括
CTLA-4抗体、PD-1抗体以及PD-L1抗体等在内的免 疫检查点抑制剂. 但值得注意的是, 这类药物针对实体 瘤时有效率却只有 $20 \% \sim 40 \%$, 疗效亟待改进. 已发现 溶瘤病毒除直接溶瘤外, 还可以通过激发抗肿瘤免疫 反应发挥抗癌作用, 因此从广义上讲, 溶瘤病毒也被 归类为一种新型的肿瘤免疫治疗药物, 而溶瘤病毒联 合免疫疗法也成为目前肿瘤治疗领域的最热点之一, 国内外已有多个相关临床试验正在开展. 2020年10月 21日，第三届“创世技”颠覆性创新榜及创新潜力榜发 布, 其中一项被誉为颠覆性的医疗创新科技, 即来自 癌症治疗领域的“CAR-T细胞负载溶瘤病毒治疗实体 肿瘤项目”。此外, 近期一项临床试验的结果显示, T-VEC 与 PD- 1 抑制剂等免疫治疗联合使用优于单纯 PD-1抑制剂. 鉴于溶瘤病毒联合免疫疗法的广阔前景, 许多大公司开始相关布局与研发. 近期PD-1抗体K药 的生产商默沙东公司, 宣布以3.94亿美元收购一家名 为Viralytics的公司, 后者的核心产品是一款已经进入 临床试验的溶瘤病毒—CVA21。

溶瘤新城疫病毒是一种来源于禽类的天然病毒, 相对于绝大多数溶瘤病毒而言, 其生物安全性极高, 并 且在人群中不存在抗体. 此外, 溶瘤新城疫病毒对肺有 较强的嗜性, 可以通过雾化途径入肺, 靶向肺癌, 这也 是溶瘤新城疫病毒的独特之处, 并已在临床试验中得 到验证. 而溶瘤新城疫病毒作为生物制剂用于治疗肿 瘤已有 60 多年的历史，目前已有多个溶瘤NDV毒株, 如HUJ和MTH/68等完成 I 或 II 期临床试验. 2003年, 中国辽宁省肿瘤医院开展了利用溶瘤NDV针对消化 道肿瘤的III期临床试验, 效果显著. 2015年, 德国批准 了NDV激活的树突状细胞疫苗(viral oncolysate-pulsed dendritic cells (VOL-DCs), Cologne, Germany)应用于 肿瘤治疗. 已完成的临床前和临床实验充分显示, 溶瘤 NDV不仅能引起癌细胞溶解, 而且能诱导长期的抗肿 瘤免疫. 利用溶瘤NDV结合免疫疗法可以有效打破传 统疗法的免疫抵抗问题, 其应用于肿瘤免疫治疗高效 且高度安全, 具有十分广阔的前景. 本课题组长期致 力于溶瘤NDV研究, 从150多病毒株中篮选鉴定出 FMW毒株(NDV/FMW), 临床前研究显示NDV/FMW 抗癌谱广, 抗癌效果强. 此外, 本课题组系统研究 了 NDV/FMW毒株的体内外杀瘤作用及机制, 发 表研究论文十多篇, 并获批一项专利 (专利号: 201810633071.3). 该专利的主要发明是DC-CIK细胞 
携带溶瘤新城疫病毒治疗肿瘤. 前期临床前研究显示, 装载溶瘤NDV的DC-CIK，其杀伤肿瘤细胞的能力得 到显著提升，这既解决了溶瘤病毒的靶向问题，又提 高了DC-CIK的杀瘤效力, 为研发下一代DC-CIK细胞 提供了方向，并可解决相关免疫细胞治疗公司的产品 升级换代问题. 此外, 目前本课题组还在探讨利用自 然杀伤(natural killer, NK)细胞或CAR-T细胞装载溶瘤 $\mathrm{NDV}$, 并与肿瘤医院共同推进溶瘤NDV临床试验的前 期工作，后期争取进入临床试验，推进恶性肿瘤的 治疗.

\section{1 溶瘤NDV简介}

\section{1 溶瘤NDV 的结构和分子生物学特征}

NDV作为一种溶瘤病毒, 是一种由双层脂质膜包 裹的RNA病毒, 属于副粘病毒科Avulavirus属的禽副粘 病毒 I 型病毒(avian paramyxovirus serotype 1, APMV$1)^{[4]}$. NDV 基因组为由 15186 个核苷酸组成的单股负链 RNA，包含 6 个开放阅读框，编码6种不同的结构蛋白: 核蛋白(nuclear protein, NP)、磷蛋白(phosphoprotein, $\mathrm{P}$ )、基质蛋白(matrix protein， M)、融合蛋白(fusion protein, F)、血凝素神经氨酸酶(hemagglutinin neura- minidase, HN)和RNA依赖性的大聚合酶蛋白(RNA-dependent large polymerase protein, L)(图1). NP, P和L蛋 白质与病毒RNA结合，形成核糖核苷酸-蛋白质复合 物, 负责病毒的复制 ${ }^{[5-9]}$. 脂质膜包围着核糖核苷酸-蛋 白质复合物, $\mathrm{M}$ 位于病毒脂质膜下一层, 参与病毒的组 装和出芽. $\mathrm{HN}$ 和 $\mathrm{F}$ 是以寡聚体形式存在的糖基化蛋白, 位于病毒膜外表面, 它们与宿主细胞的脂质双层膜一 起构成病毒的外壳, 参与病毒感染宿主细胞的过 程 ${ }^{[10,11]}$.

NDV 主要感染禽类, 不感染人在内的哺乳动物. NDV感染宿主细胞的主要过程如下: (i ) 由病毒表面 $\mathrm{HN}$ 和 F糖蛋白与含唾液酸的宿主细胞表面蛋白结 合 ${ }^{[12,13]}$, 触发 $F$ 蛋白的构象变化, 释放融合肽, 促进病毒 包膜和细胞质膜的融合, 病毒颗粒通过内吞作用使具 有附着颗粒的相邻细胞形成合胞体 ${ }^{[8,13,14]}$. 病毒基因组 进入宿主细胞的细胞质, $\mathrm{M}$ 蛋白从细胞质中的核糖核 酸蛋白复合物中分离, $\mathrm{P}$ 和L蛋白形成聚合酶复合物, 启动病毒RNA的转录 ${ }^{[12,15]}$. (ii) 病毒基因组在宿主细 胞质中复制 ${ }^{[16]}$. 首先, 基因组 $\operatorname{ssRA}(-)$ 在细胞质中转 录为mRNA, 翻译成不同的结构蛋白. 合成的一个全长 RNA分子 $\operatorname{ssRNA}(+)$, 被用作病毒基因组扩增的模板, 复制出完整负链病毒RNA分子, 随后产生的病毒后代

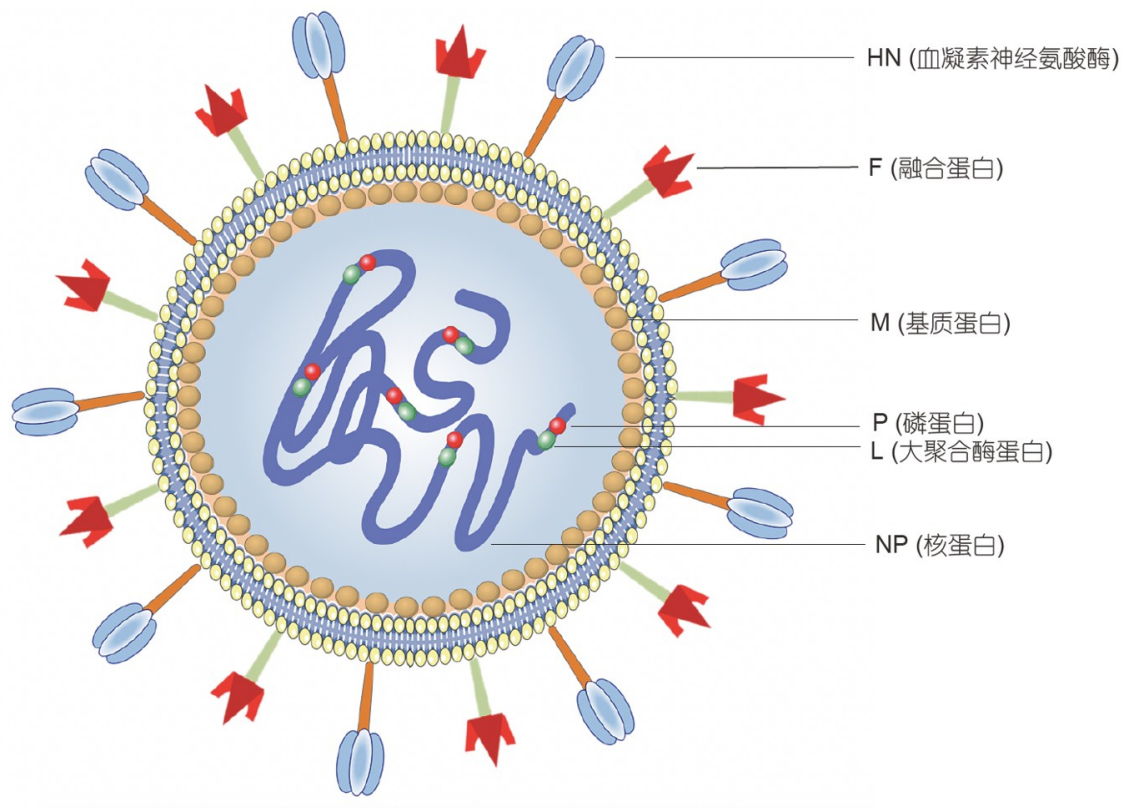

图 1 NDV的分子结构. NDV结构蛋白由NP, P, L, M, HN和F蛋白构成, 其中NP, P和L与病毒RNA结合形成核糖核蛋白复合物 Figure 1 Molecular structure of NDV. The structural proteins of NDV consist of the NP, P, L, M, HN, and F proteins; among these, the NP, P, and L proteins associate with viral RNA to form the ribosome-nuclear protein complex 
通过出芽被释放到细胞外, 引发宿主细胞的新一轮感 染 ${ }^{[17]}$. 溶瘤NDV在肿瘤细胞中的增殖过程与NDV感染 禽类细胞类似.

\section{2 溶瘤NDV的研究简史}

使用NDV来改善癌症治疗已有 50 多年. 20 世纪 50 年代, 人们发现NDV具有溶瘤特性. 第一例使用NDV 治疗人类癌症(急性白血病)的报道发表于 1964 年 ${ }^{[18]}$, 该患者在使用NDV后经历了短暂的抗白血病作用和 临床症状的短暂缓解. 1965年, 来自亚特兰大的Cassel 和 Garret ${ }^{[19]}$ 首次使用NDV(溶瘤毒株 73T) 作为抗肿瘤 制剂。人们在 20 世纪 60 和 70 年代进行了溶瘤NDV的一 系列开创性研究, 然后出现了一个缺口, 当时癌症研究 科学家对细胞抑制药物的开发和对肿瘤基因及肿瘤抑 制基因的研究更感兴趣. 20 世纪 90 年代和 21 世纪初, 人 们对溶瘤病毒和NDV的研究兴趣有所回升 ${ }^{[20]}$. 50多年 的NDV临床应用证明了溶瘤NDV作为生物制剂的高 度安全性。2003年，中国辽宁省肿瘤医院分别使用 NDV修饰的自体瘤苗和NDV毒株治疗消化道肿瘤患 者，其生存期显著延长(7年vs. 4.46年). 2015年，溶瘤 NDV的临床应用实现了一个重要里程碑, 即德国IOZK 首次获得了生产NDV致敏树突状细胞疫苗的GMP证 书. 根据良好生产规范(Good Manufacturing Practice, GMP)成功生产NDV，NDV不仅可以从鸡胚囊中产生， 而且可以从细胞培养中产生. 表 1 概述了 50 年来对 NDV的研究, 包括基础研究和临床应用.

\subsection{NDV 的溶瘤机制}

人类各种肿瘤细胞, 如肝癌 ${ }^{[67]}$ 、胶质母细胞瘤 ${ }^{[68]}$ 和淋巴瘤 ${ }^{[69]}$ 均对NDV敏感. 此外, 由于NDV的RNA转 录和翻译与细胞增殖无关，NDV可以靶向感染肿瘤干
细胞、休眠肿瘤细胞和X射线照射的肿瘤细胞 ${ }^{[70]}$.

NDV选择性感染肿瘤细胞并诱导肿瘤细胞死亡 (溶瘤). NDV溶瘤的初始阶段是病毒介导肿瘤细胞的 直接溶瘤，随后第二阶段是溶瘤后引起免疫反应，激 活机体免疫系统，将“冷肿瘤”转变为“热肿瘤”. NDV 的溶瘤机制主要包括以下5个方面: (i) NDV选择性 感染肿瘤细胞, 在肿瘤细胞中复制而裂解肿瘤细胞. (ii) 释放肿瘤抗原, 激活机体免疫应答, 先天性免疫 和适应性免疫反应, 直接激活免疫细胞, 如巨噬细胞、 针对抗原的NK细胞和细胞毒性 $\mathrm{T}$ 淋巴细胞 ${ }^{[71,72]}$. (iii) 包膜蛋白参与溶瘤作用. (iv) 调亡通路促进溶瘤作用. ( v ) NDV诱导肿瘤免疫原性细胞死亡、细胞焦亡、 坏死和自噬(图2).

(1) NDV选择性感染肿瘤细胞和复制. NDV感染 细胞主要包括两个步骤: ( i ) 细胞结合、膜融合、病 毒基因组的转导和病毒基因的转录 ${ }^{[73]}$; (ii) 利用新产 生的核衣壳进行病毒复制 ${ }^{[17,74]}$. 所有RNA病毒的复制 都涉及双链RNA(double-stranded RNA， dsRNA)的形 成, 这种外来结构激活了基于 I 型干扰素(如IFN- $\alpha$ 和 - $\beta$ )的重要细胞防御系统. 肿瘤细胞的突变往往会削弱 干扰素系统, 从而使细胞增殖不受抑制, 并具有调亡抗 性. 在非禽类宿主(如小鼠或人)中, 这种突变允许NDV 选择性感染肿瘤细胞，在肿瘤细胞中复制，而NDV感 染非禽类宿主的正常细胞通常不会进入第二步 ${ }^{[17,75]}$. NDV在人类肿瘤细胞中的复制速度比在大多数正常 细胞中的复制速度快 10000 倍.

NDV毒株可分为裂解型病毒和非裂解型病毒. 这 两种病毒都能杀死癌细胞, 但裂解型病毒有可能更快 地杀死癌细胞, 因为它们破坏了被感染细胞的质膜. 此外, 裂解型病毒表现出多环复制, 而非裂解型病毒 仅表现出单环复制. NDV诱导的调亡依赖于TNF相关

表 150 年来对NDV溶瘤的研究

Table 1 Fifty years of research on NDV

\begin{tabular}{cccc}
\hline 时间 & 基础研究 & 临床研究 & 参考文献 \\
\hline $1960 \mathrm{~s}$ & 病毒溶瘤, 溶瘤后免疫 & 单病例的观察 & {$[18,21,22]$} \\
$1970 \mathrm{~s}$ & 研究溶瘤机制, 病毒溶瘤液 & 术后病毒疫苗接种 & {$[19,23 \sim 27]$} \\
$1980 \mathrm{~s}$ & ATV-NDV活细胞疫苗 & 使用病毒溶瘤液疫苗接种 & {$[27 \sim 37]$} \\
$1990 \mathrm{~s}$ & 基因组测序, 免疫细胞激活机制研究 & ATV-NDV吸入疫苗接种的 I II 期试验 & {$[38 \sim 46]$} \\
$2000 \mathrm{~s}$ & 重组改良NDV毒株 & 全身剂量 II/III期试验 & {$[31,32,47 \sim 54]$} \\
$2010 \mathrm{~s}$ & 高质量(GMP)病毒生产 & 病毒溶瘤液和DC细胞(VOL-DC)联合使用 & {$[44,45,55 \sim 66]$} \\
\hline
\end{tabular}




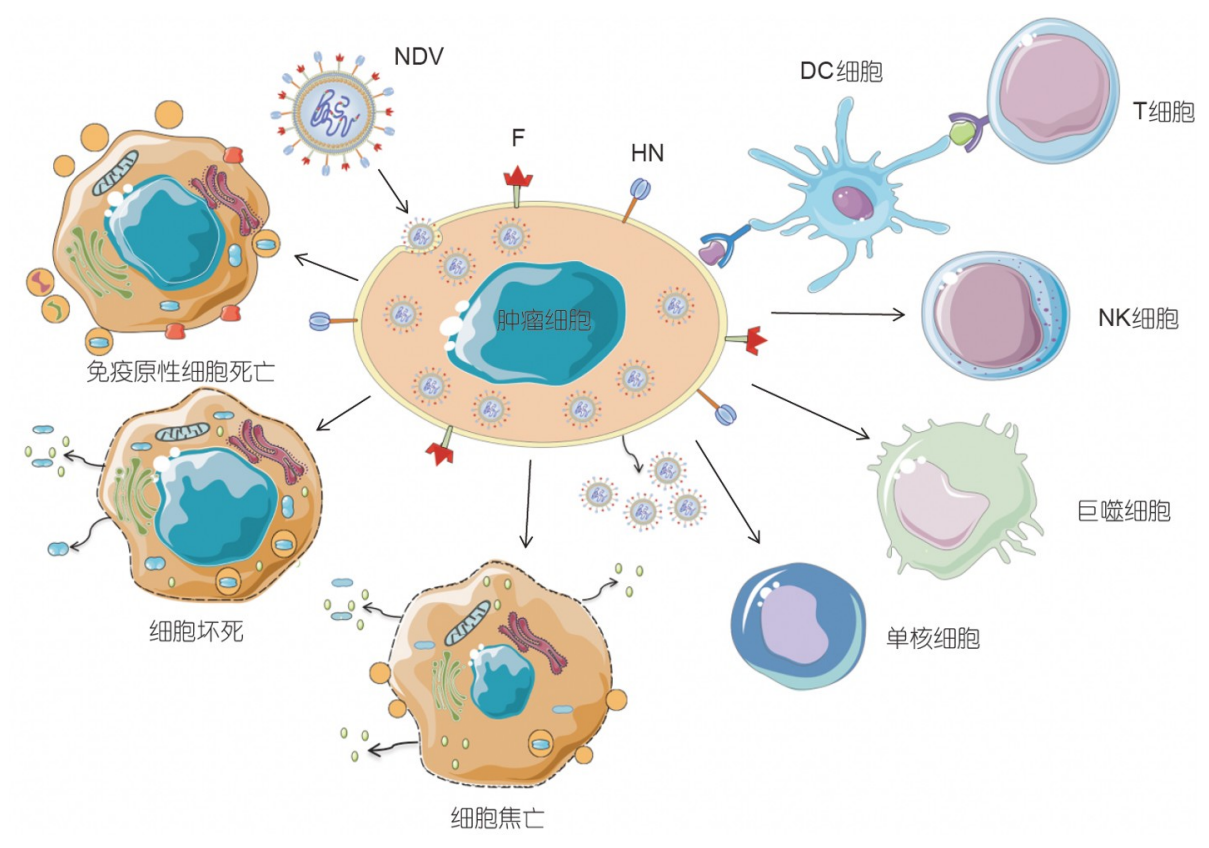

图 2 NDV感染肿瘤细胞的抗肿瘤机制. NDV通过内吞作用进入肿瘤细胞的细胞质, 刺激宿主的先天性免疫反应, 激活NK细 胞、单核细胞和巨噬细胞, 促进适应性免疫反应. NDV可通过诱导肿瘤细胞免疫原性死亡、细胞坏死和细胞焦亡等杀伤肿瘤 细胞

Figure 2 The antitumor mechanism of NDV infecting tumor cells. NDV enters the cytoplasm of tumor cells via endocytosis, then stimulates the innate immune response, activating natural killer cells, monocytes, and macrophages, thereby promoting the adaptive immune response. Moreover, NDV kills tumor cells by triggering immunogenic cell death, necrosis, and pyroptosis

调亡诱导配体(TNF-related apoptosis-inducing ligand, TRAIL)的上调和caspase的激活. 该病毒通过内源性和 外源性caspase依赖的细胞死亡途径介导其溶瘤作用.

已确定的在非禽类宿主中, NDV选择性在肿瘤中 复制和溶瘤的机制包括: ( i ) 肿瘤细胞中抗病毒信号 通路激活的缺陷 ${ }^{[76] ;}$; (ii) 肿瘤细胞中 I 型IFN信号通 路的缺陷 ${ }^{[77]}$; (iii) 肿瘤细胞中调亡通路的缺陷 ${ }^{[68]}$; (iv) Ras信号激活和Rac1蛋白的表达 ${ }^{[78]}$.

(2) NDV直接激活免疫细胞. (i ) 激活NK细胞: NDV的病毒表面蛋白HN，可诱导NK细胞活化. 激活 $\mathrm{NK}$ 细胞有两种受体 NKp46和NKp44, 分别与信号链

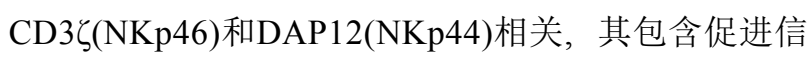
号转导的免疫受体酪氨酸基激活基序(immunoreceptor tyrosine-based activation motif, ITAM) 结构域 ${ }^{[79]}$.

(ii) 激活单核细胞和巨噬细胞: 与NDV接触后, 单核细胞被激活, 通过TRAIL产生杀瘤作用 ${ }^{[00]}$. NDV 激活的巨噬细胞不仅在体外具有抗肿瘤活性，而且在 体内也具有抗肿瘤活性 ${ }^{[80,81]}$. NDV感染的巨噬细胞通 过激活 $\mathrm{NF \kappa B}$ 产生一氧化氮 $(\mathrm{NO})^{[82]}$, 诱导肿瘤细胞调 亡. 在人类肿瘤淋巴细胞中, NO介导的、CD95依赖的
和独立的调亡需要半胱天冬酶激活 ${ }^{[83]}$.

(iii) 激活树突状细胞: 当暴露于 $\mathrm{NDV}$ 等微生物刺 激物时, DC激活 NF- $\mathrm{kB}$, 诱导表达促炎细胞因子, 促进 $\mathrm{CD}^{+} \mathrm{Th} 1$ 反应和 $\mathrm{CD} 8^{+}$效应 $\mathrm{T}$ 细胞的产生. NDV通过刺 激DC分泌大量IFN- $\alpha$, 介导先天性免疫和适应性免疫 之间的联系, DC还可通过促进T细胞分泌IFN- $\gamma$ 和IL-2, 触发有效的抗肿瘤免疫 ${ }^{[84]}$.

(3) NDV包膜蛋白参与溶瘤作用 ${ }^{[85]}$. 研究发现, NDV的多种蛋白可以影响感染细胞, 蛋白序列分析显 示 $M, L$ 和 F 具有一个与促凋亡因子 Bcl-2同源的BH-3结 构域. 其中 HN是重要的免疫原性蛋白和毒力因子, HN 诱导人外周血单个核细胞释放 I 型IFN并上调TRAIL 的表达 ${ }^{[86]}$. 表达NDV HN蛋白的新型溶瘤腺病毒 (Ad-hTERTp-E1a-HN), 可以选择性抑制食管癌EC-109 细胞并抑制小鼠肿瘤生长. 此外, 有研究表明, NDV AF2240株M蛋白通过其BH-3结构域与Bax结合, 促进 $\mathrm{Bax}$ 从细胞质向线粒体膜转移, 从而激活其固有的调 亡通路 ${ }^{[87]}$.

(4) 调亡通路参与NDV的溶瘤作用 ${ }^{[85]}$. 多项研究 表明, NDV的溶瘤作用与细胞的多种调亡途径有关, 
NDV诱导细胞调亡需要病毒复制和调亡蛋白的表达.

外源性调亡途径主要由死亡受体 Fas及其配体 FasL和TRAIL介导. 例如, 由于Fas的过表达, 重组减毒 株rNDV-B1/Fas在体外和体内均表现出溶瘤能力的增 强. 与野生型病毒相比, 重组病毒LaSota-TRAIL组的 TRAIL表达增加3倍. 在小鼠实验中, LaSota-TRAIL组 表现为出生存率显著提高和肿瘤复发减少. 其他研究 表明，调亡抑制蛋白 survivin的上调可延长NDV AF2240株感染的人乳腺癌细胞的生存能力, 并增加病 毒蛋白的合成和病毒复制 ${ }^{[88]}$.

(5) NDV诱导肿瘤免疫原性细胞死亡. 免疫原性细 胞死亡(immunogenic cell death, ICD) 是溶瘤NDV介导 免疫治疗的一个重要新方式. 经典的生理调亡是非免 疫原性的. NDV诱导肿瘤细胞ICD的特征包括内质网 应激反应、免疫原性调亡、坏死和自噬. 这种特征使 蛋白质合成终止，随后暴露于钙蛋白、热休克蛋白和 病毒蛋白HN和F. 本课题组与合作者先后报道了溶瘤 NDV 在恶性黑色素瘤及前列腺癌中引起免疫原性细 胞死亡 ${ }^{[89,90]}$.

（6）溶瘤NDV诱导坏死、自噬和铁死亡.NDV感 染肿瘤细胞后, HN和F蛋白在宿主细胞表面积累, 促进 细胞合胞体的形成, 导致细胞间融合, 最终引发细胞坏 死、合胞体解体、内容物释放和炎症反应. caspase 8 也可通过细胞TLR和TNF家族激活细胞坏死.

NDV的HN和F蛋白在肺腺癌细胞(A549)中迅速诱 导合胞体形成并启动稳定的自噬, 协同诱导自噬体与 溶酶体融合, 实现细胞降解. 自噬有利于NDV感染早 期肿瘤细胞的病毒复制, 通过调节调亡延长细胞生命 周期. 有报道称, 线粒体自噬通过打破肺腺癌细胞固 有的调亡调节途径来促进NDV的复制 ${ }^{[91]}$. 本课题组 ${ }^{[92]}$ 前期报道了NDV感染对肿瘤细胞凋亡和自噬的影响, 发现NDV引起肺癌细胞凋亡依赖于p38/MAPK通路. 此外, 本课题组 ${ }^{[93]}$ 首次报道溶瘤NDV引起肿瘤细胞自 噬, 并影响病毒在肿瘤细胞中的复制. 本课题组 ${ }^{[94]}$ 进 一步的研究发现, NDV能诱导耐顺铂肺癌细胞调亡, 调控自噬促进NDV杀伤耐药肺癌细胞能力, 自噬调节 剂氯喹和雷帕霉素可显著增强细胞自噬和NDV对小 鼠A549细胞的溶瘤作用, 这一发现为探索自噬调控因 子和NDV联合使用的抗肿瘤策略提供了新的思路.

此外, 本课题组 ${ }^{[95]}$ 参与的一项由丁铲教授主持的 研究首次证实NDV引起肿瘤细胞铁死亡, 这一发现表
明，溶瘤NDV的杀瘤作用机制及其复杂，而溶瘤机制 的多样性有助于赋予NDV更广的溶瘤谱及对放化疗 抵抗肿瘤的杀伤力. 综上所述, 溶瘤NDV通过多条途 径及方式抑制肿瘤生长, 发挥其杀瘤作用, 为充分挖 掘溶瘤NDV的临床应用价值提供了理论基础.

\section{4 使用NDV作为溶瘤制剂的优势}

与其他病毒家族(如逆转录病毒或DNA病毒)相比, 使用NDV作为溶瘤制剂具有以下优势. (i) NDV属于 禽类病毒, 感染家禽和鸟类, 对人无致病性, 使用更安 全; 溶瘤NDV静脉注射食蟹猴, 未发生血液或生化指 标异常. (ii) NDV是单股负链RNA病毒, 它的复制过 程发生在细胞质中, 从RNA到RNA, 不存在DNA阶段, 这种细胞质复制使病毒独立于宿主细胞DNA复制机 制, 不能和宿主基因组整合, 也不与人类自身病毒发生 重组. (iii) NDV体内给药方式多样, 可通过瘤内注射、 静脉注射、鼻腔吸入、喷雾等多种方式, 使用方便, 癌 症患者甚至可以耐受相对高剂量的NDV. 临床试验报 道, NDV感染产生的副作用较少, 通常表现为轻度流 感样症状, 如疲劳、头痛、发烧、虚弱. 即便偶尔有 些患者出现短暂的血小板减少或中性粒细胞减少, 这 些副作用也只是暂时的, 在接种NDV疫苗后的一到两 天内就会消失. (iv) NDV减毒疫苗具有高滴度的鸡胚 生长特性, 生产成本低廉. ( v ) NDV可以选择性在肿瘤 中高效复制, 不会在正常细胞中复制. 此外, NDV具有 在非增殖性肿瘤细胞中复制的能力, 由于病毒在细胞 质中的复制与细胞增殖无关, NDV在肿瘤细胞或休眠 的肿瘤细胞中复制, 可能不会受化疗或放疗的影响, 并且可以在耐调亡的肿瘤细胞、耐缺氧的肿瘤细胞和 耐干扰素肿瘤细胞中复制. (vi) 除了NDV直接的溶瘤 作用, 它还可以促进免疫系统的激活, 发挥抗肿瘤活 性. 一方面, NDV可直接刺激免疫细胞, 将肿瘤微环境 的免疫抑制状态转变为免疫激活状态, 如显著增加肿 瘤微环境中的 $\mathrm{CD} 4^{+}$和 $\mathrm{CD} 8^{+} \mathrm{T}$ 细胞浸润, 上调免疫检查 点分子CTLA-4和PD-1等, 这为使用NDV和免疫检查 点抑制剂结合打破免疫抵抗, 提高免疫检查点抑制剂 疗效提供可能. 另一方面, NDV感染肿瘤细胞可以诱 导IFN- $\alpha$, IFN- $\beta$, TNF- $\alpha$ 和IL- 1 等细胞因子的产生. 因 此, 肿瘤外有大量的免疫细胞被诱导浸润到肿瘤中, 激活的非特异性免疫细胞可以杀死和吞噬感染的尚未 溶解的肿瘤细胞或对病毒溶瘤有抵抗的肿瘤细胞. 


\subsection{NDV治疗癌症的临床应用}

NDV应用于临床治疗癌症已有 50 多年，作为一种 溶瘤制剂, 其研发经历了以下几个阶段 ${ }^{[96]}$.

（1）20世纪60 70年代: 使用NDV制剂进行术后治 疗. 1965年, Cassel等人首次将NDV 73T株直接注射到 宫颈癌患者的肿瘤区域. 结果显示, 肿瘤广泛脱落, 锁 骨上淋巴结转移减少，初步证实了NDV的抗肿瘤潜力 和安全性. 此后, 他们提出了使用自体或异体病毒溶瘤 液作为疫苗的策略，并在 II 期恶性黑色素瘤术后治疗 中, 采用NDV溶瘤液作为辅助免疫治疗. 对 83 名接受 治疗的患者进行的 10 年随访显示， $60 \%$ 以上的患者仍 然存活, 并且没有复发. 在德国, Kirchner等人对 208 名 局部晚期肾细胞癌患者进行了一项类似的二期研究, 研究对象为自体NDV修饰的肿瘤裂解物疫苗, 与仅接 受手术治疗的类似患者相比，接受术后NDV修饰的自 体肿瘤裂解物疫苗治疗的患者, 无病生存率有所提高.

（2）20世纪90年代 21世纪初: 使用自体肿瘤细胞 NDV活疫苗(autologous tumor-cell NDV, ATV-NDV)进 行术后治疗. 为了提高NDV修饰肿瘤疫苗的免疫原性, 德国海德堡癌症研究中心(Deutsches Krebsforschungszentrum, DKFZ)提出了一种新概念, 即开发一种病毒 感染经辐照的自体肿瘤细胞活细胞疫苗(ATV-NDV), 并在各种转移性动物肿瘤模型系统中成功地验证了该 概念. 在七种不同类型的癌症(乳腺癌、结肠癌、直肠 癌、肾癌、头颈癌、胰腺癌和多形性胶质母细胞瘤) 中使用了这种疫苗，结果表明，使用高质量的ATVNDV疫苗可明显改善患者的预后生存期，且未观察到 严重副作用。该临床试验结果表明，对肿瘤患者接种 ATV-NDV是可行和安全的.

(3) 21 世纪初, 溶瘤NDV的系统应用研究. 在德国, Csatary等人通过静脉注射减毒溶瘤NDV株(MTH-68/ $\mathrm{H})$ ，对四种高级别胶质瘤进行了一项选定的病例系列 研究. 此外, 在美国, Wellstat Biologics将溶瘤NDV株 PV701应用于标准治疗无效的晚期癌症患者. 79名晚 期癌症患者接受了递增剂量的病毒注射，注射 $12 \times 10^{9} \sim 12 \times 10^{10}$ 感染颗粒(斑块形成单位 $/ \mathrm{m}^{2}$ ) 的剂量具 有良好的耐受性.

（4）结合溶瘤病毒修饰疫苗(ATV-NDV)和双特异 性抗体共刺激. 使用ATV-NDV疫苗进行的大多数临床 试验显示, 约 $30 \%$ 的受试者总生存期得到改善, 其余约
$70 \%$ 必须被视为免疫无应答者. $\mathrm{T}$ 细胞无反应性是癌症 患者的一个主要问题, 并且这通常是由于共刺激不足 所致. 而向ATV-NDV疫苗中添加具有双特异性的 $\mathrm{NDV}$ 特异性单链抗体 (双特异性单链抗体, bsAb), 可 以增强疫苗ATV-NDV中的T细胞共刺激信号. 研究表 明, ATV-NDV肿瘤疫苗, 带有附加的抗-HN-抗-CD3 $(\alpha \mathrm{HN}-\alpha \mathrm{CD} 3)$ 和抗-HN-抗-CD $28(\alpha \mathrm{HN}-\alpha \mathrm{CD} 28) b s \mathrm{bb}$ ，在 体外刺激人外周血单个核细胞(peripheral blood mononuclear cell, PBMC), 对人肿瘤细胞具有强而持久的抗 肿瘤作用. Schirrmacher等人在ATV-NDV的表面添加 了一种bsHN-CD28，构建的bsHN-CD28通过一只臂与 NDV的HN分子结合, 另一只臂针对 CD28, CD28是T 细胞上传递共刺激信号的重要分子. 对14例晚期大肠 癌患者使用ATV-NDV疫苗加bsHN-CD28治疗后, 未发 生严重不良事件。这项研究表明，三组分疫苗是安全 的，而ATV-NDV的表面添加T细胞共刺激信号，可以 重新激活在晚期癌症中可能被激活的 $\mathrm{T}$ 细胞. 表2 总结 了溶瘤NDV治疗癌症的临床应用研究.

\section{NDV与肿瘤免疫治疗}

\subsection{NDV 激活抗肿瘤免疫应答}

NDV选择性感染肿瘤细胞，肿瘤细胞裂解后释放 病原体相关分子(pathogen-associated molecular patterns, PAMPs)、危险相关分子(damage-associated molecular patterns，DAMPs)、肿瘤相关抗原(tumourassociated antigens, TAAs)、细胞因子(cytokines, CKs) 等物质, TAAs募集抗原提呈细胞(antigen-presenting cell, APC), 活化的APC将TAA呈递给杀伤性T细胞, 引 起免疫应答, 如图3所示. NDV感染引起肿瘤微环境改 变, 并激活先天性和适应性抗肿瘤免疫反应 ${ }^{[97]}$.

(1) NDV激活天然抗肿瘤免疫应答. I 型IFN信号 通过招募固有细胞(包括NK细胞和APC)、上调细胞 黏附、主要组织相容性复合体(major histocompatibility complex, MHC) 和共刺激分子以及启动抗原特异性 T细胞来激活固有免疫和适应性免疫. I 型IFN信号的 激活是癌症免疫治疗的关键途径之一, 肿瘤细胞通常 具有受损的 I 型IFN信号，这也是导致肿瘤细胞对 NDV 感染敏感性增加的主要机制之一. 注射NDV的小 鼠肿瘤的转录谱显示 I 型IFN反应相关基因和一系列 细胞因子、趋化因子上调, 它们介导先天性和适应性 
表 2 溶瘤NDV的临床应用

Table 2 Clinical applications of oncolytic Newcastle disease virus

\begin{tabular}{|c|c|}
\hline NDV毒株类型 & 临床试验 \\
\hline 溶瘤毒株73 T & WA Cassel和Murray博士(1977年)对 II 期黑色素瘤患者(83例)应用NDV溶瘤疫苗进行术后治疗 \\
\hline 溶瘤毒株MTH/68 & 晚期化疗难治性癌症的系统治疗: Cstatary和Eckhardt(1993)进行的 II 期试验 \\
\hline 减毒毒株HUJ & $\begin{array}{c}\text { 14例胶质母细胞瘤(glioblastoma, GBM)的系统治疗: Freeman和Zakay-Rones(2006年)在患者体内进行了3 } \\
\text { 个周期的550亿单位的病毒感染试验 }\end{array}$ \\
\hline 溶瘤毒株PV71 & Pecora和Lorence(2002年、2003年)对耐标准治疗的晚期实体癌患者(113例)静脉注射病毒的 I 期试验 \\
\hline 减毒毒株Ulster & $\begin{array}{c}\text { 使用NDV感染经辐照的自体肿瘤细胞活细胞疫苗(ATV-NDV)进行术后治疗: } \\
\text { (1) Ahlert和Schirrmacher(1997)研究的早期乳腺癌(63例)、转移性乳腺癌(27例)和转移性卵巢癌(31例); } \\
\text { (2) Ockert和Schirrmacher(1996)对晚期大肠癌患者(57例)进行的 II 期试验; } \\
\text { (3) Steiner和Herold-Mende(2004年)在GBM患者中(23例接种疫苗的患者和在同一时间内来自同一诊所的 } \\
\text { 87例未接种疫苗的患者)进行的 II 期试验; } \\
\text { (4) Karcher和Dyckhoff(2004年)对III期和IV期头颈鳞状细胞癌患者(20例)进行的 I / II 期试验 }\end{array}$ \\
\hline 减毒毒株Ulster & $\begin{array}{c}\text { Schulze和Schlag(2009)进行的前瞻性随机 II/III期试验, 旨在研究ATV-NDV疫苗在大肠癌肝转移肝切除术 } \\
\text { (51例)后作为三级预防方法的有效性 }\end{array}$ \\
\hline NDV修饰的自体瘤苗和NDV毒株 & 辽宁省肿瘤医院 2003年III期试验, 治疗消化道肿瘤 \\
\hline
\end{tabular}

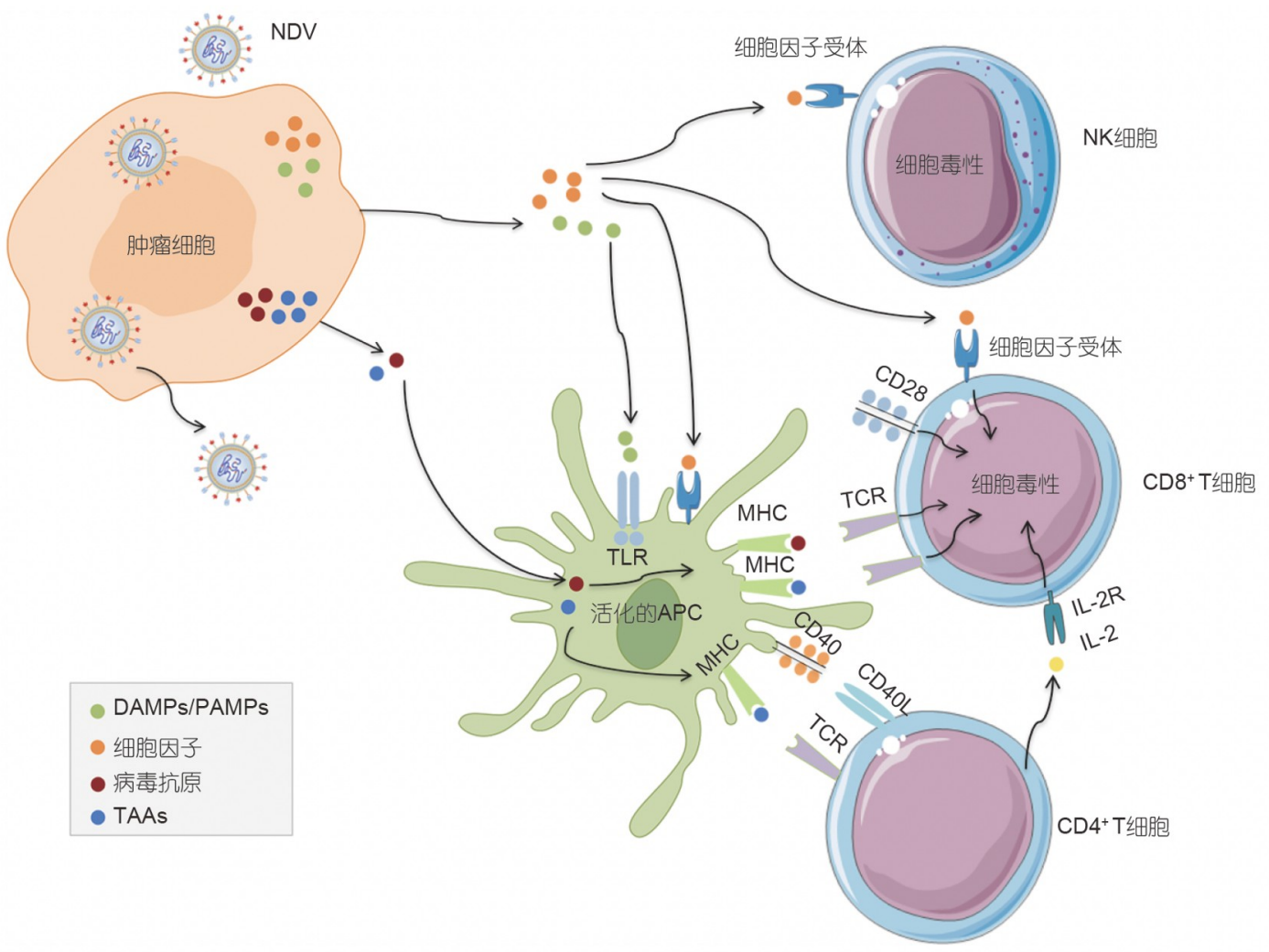

图 3 溶瘤NDV引起抗肿瘤免疫反应

Figure 3 Oncolytic NDV induces the antitumor response

免疫细胞的募集和增殖.

除了激活肿瘤细胞的 I 型IFN信号外，NDV产生 的炎症环境导致天然效应细胞和适应性免疫细胞的募
集，这些细胞有助于抗肿瘤免疫。特别是瘤内注射 NDV可导致NK细胞明显浸润肿瘤．NDV感染还导致 髓样细胞的募集, 髓样细胞在吞噬和抗原呈递中起着 
重要作用.

(2) NDV激活适应性抗肿瘤免疫应答. NDV感染 后激活由 I 型IFN信号介导的先天性抗肿瘤免疫反应, 为刺激适应性抗肿瘤免疫提供了最佳条件. 炎症介质 的分泌导致髓样细胞和淋巴样细胞向肿瘤微环境募 集. 一个关键效应细胞群是 $\mathrm{DC}$ 和抗原特异性 $\mathrm{CD} 8^{+} \mathrm{T}$ 细 胞的启动. NDV感染可通过调亡、坏死或自噬、免疫 原性细胞死亡，导致肿瘤微环境中病毒和肿瘤相关抗 原和碎片的释放，抗原呈递 $\mathrm{DC}$ 在摄取这些抗原以及 PAMP和DAMPs时被激活和成熟. 抗原呈递DC产生的 白细胞介素(IL-12)与肿瘤微环境中的 I 型IFN信号协 同作用，导致MHCI类和 II 类分子、细胞黏附分子和 共刺激分子的上调，所有这些都促进了APCs对 T细胞 的启动.

NDV诱导的肿瘤抗原特异性 $\mathrm{CD} 8^{+} \mathrm{T}$ 细胞反应的 证据来自于双侧侧翼同基因肿瘤模型的研究, 将NDV LaSota毒株注射到单个侧翼肿瘤, 由于病毒在注射肿 瘤部位复制的限制，这种模型允许评估局部和远处的 免疫效应. 有趣的是, 注射和未注射NDV的肿瘤内 $\mathrm{CD} 4^{+}$和 $\mathrm{CD} 8^{+} \mathrm{T}$ 细胞浸润显著增加, 在注射病毒的肿瘤 和远处肿瘤的肿瘤浸润性 $\mathrm{T}$ 细胞上观察到许多免疫检 查点分子的上调, 包括CTLA-4和PD-1. 这些发现表明, 联合NDV和免疫检查点抑制剂有可能打破免疫抵抗. 事实上, 与单独治疗相比, NDV与全身抗CTLA-4、抗 PD-1或抗PD-L1的联合治疗具有更好的抗肿瘤疗效, 延长动物存活时间，这一效果在多种肿瘤模型中均有 体现．这些研究表明，NDV瘤内治疗可以提高免疫检 查点抑制剂的系统疗效.

\subsection{NDV与免疫治疗联合治疗肿瘤的研究和临床 应用}

基于NDV独特的抗肿瘤特性及其与患者免疫系
统的积极相互作用，从治疗原则来看，将NDV与多模 式免疫治疗相结合, 能充分调动机体的抗肿瘤反应. 表3总结了NDV通过多模式免疫治疗的研究和临床应 用情况.

\section{NDV临床治疗的限制和挑战}

在过去的 60 年中, 多项临床前研究和溶瘤NDV反 复证明了其作为溶瘤制剂和免疫治疗制剂的优势和巨 大潜力. 然而, 有不少问题有待解决, 其中一个关键问 题是靶向给药. 目前还没有针对患者使用病毒的方式 和剂量的优化标准方案和免疫检查点抑制剂联合治疗 的最佳组合.

此外, 在治疗效果方面, 实体肿瘤的细胞外基质和 其他屏障可能干扰和减缓病毒感染和传播，从而减少 溶瘤效果. 另外, 外源基因插入NDV基因组有利弊: 一 方面，外源基因可能增强NDV的抗肿瘤作用; 另一方 面, 外源基因的插入可能会影响病毒的复制.

\section{4 未来展望}

如何利用溶瘤病毒, 提升抗肿瘤的有效性, 是研究 人员需要考虑的重要科学问题. 溶瘤NDV作为肿瘤治 疗的一种新型疗法, 具有许多先决条件: 高安全性、较 化疗或放疗低的副作用、选择性强溶瘤作用、强 的 I 型IFN反应和广泛的免疫刺激作用. 在未来, 如何 利用溶瘤NDV与其他治疗方式结合以介导更强的抗 肿瘤作用, 是值得思考和研究的主题. 本文认为多模式 联合治疗是治疗肿瘤的理想选择，通过将溶瘤NDV与 热疗、已批准的化疗药物和小分子、免疫调节剂、树 突状细胞、活化的 T细胞、双特异性抗体或NDV疫苗 (如ATV-NDV)结合，多模式联合治疗具有广阔的应用

表 3 NDV结合免疫治疗疗法的研究和临床应用

Table 3 Research and clinical applications of Newcastle disease virus combined with immunotherapy

\begin{tabular}{cc}
\hline 联合疗法方式 & 临床试验 \\
\hline NDV 结合免疫检查点抑制剂 & $\begin{array}{c}\text { Gayathri等人首次构建了表达免疫检测点抑制剂抗体的重组NDV(rNDV-anti-PD1和rNDV-anti-PDL1), 建 } \\
\text { 毒联合CTLA4阻滞剂治疗小鼠黑色素瘤的模型. 与单药治疗相比, 联合治疗增强了抗肿瘤作用, } \\
\text { 延长了生存时间 }\end{array}$ \\
NDV结合免疫调节剂 & $\begin{array}{c}\text { 表达干扰素和促炎细胞因子的NDV, 如rNDV-INF, rNDV-IL24, rNDV-IL2和rNDV-IL12. 在动物体内和体外 } \\
\text { 实验中, 相比野生型NDV, 重组NDV均表现出更强的抗肿瘤作用 }\end{array}$ \\
ATV-NDV装载DC细胞 & 1例伴有骨转移的前列腺癌患者经治疗后病情缓解 \\
\hline
\end{tabular}


前景, 有利于克服传统疗法的临床障碍. 尽管溶瘤新城 疫病毒的生物安全性高, 在开展溶瘤新城疫病毒的临 床试验之前，还需考虑如何制备临床级溶瘤新城疫病 毒制剂的问题. 由于需要从鸡胚尿囊液制备新城疫病 毒原液, 该原液含有异种蛋白, 需要深度纯化, 才能获 得临床级病毒制剂，这也增加了大规模制备溶瘤新城 疫病毒的难度.
毫无疑问, 溶瘤NDV的前方充满机遇. 一方面, 可 以通过对免疫机制的进一步了解，开发出更好的溶瘤 $\mathrm{NDV}$, 进一步拓展它的潜力; 另一方面, 应将这一充满 潜力的工具与现有疗法进行结合, 利用其对免疫反应 的影响能力, 实现更有效的抗肿瘤作用. 随着免疫系 统抗肿瘤机制的不断阐明, 溶瘤NDV有望为免疫疗法 提供更广阔的应用平台.

\section{参考文献}

1 Huang Y, Mao Q, He J, et al. Fusions of tumor-derived endothelial cells with dendritic cells induces antitumor immunity. Sci Rep, 2017, 7: 46544

2 Inoda S, Morita R, Hirohashi Y, et al. The feasibility of Cep55/c10orf3 derived peptide vaccine therapy for colorectal carcinoma. Exp Mol Pathol, 2011, 90: 55-60

3 Xu-Monette Z Y, Zhang M, Li J, et al. PD-1/PD-L1 blockade: have we found the key to unleash the antitumor immune response? Front Immunol, 2017,8

4 Amarasinghe G K, Ayllón M A, Bào Y, et al. Taxonomy of the order Mononegavirales: update 2019. Arch Virol, 2019, 164: 1967-1980

5 Liu T, Song Y, Yang Y, et al. Hemagglutinin-neuraminidase and fusion genes are determinants of NDV thermostability. Vet Microbiol, 2019, 228: 53-60

6 Marcos F, Ferreira L, Cros J, et al. Mapping of the RNA promoter of Newcastle disease virus. Virology, 2005, 331: 396-406

7 Lamb R, Parks G. Paramyxoviridae: the viruses and their replication. In: Knipe D M, Howley P M, eds. Fields Virology. Philadelphia: Lippincott Williams \& Wilkins, 2007. 1449-1496

8 Sinkovics J G, Horvath J C. Newcastle disease virus (NDV): Brief history of its oncolytic strains. J Clin Virol, 2000, 16: 1-15

9 Leighton F A, Heckert R A. Newcastle disease and related avian paramyxoviruses. Infect Dis Wild Birds, 2008, 1: 1-16

10 Fournier P, Zeng J, Von Der Lieth C W, et al. Importance of serine 200 for functional activities of the hemagglutinin-neuraminidase protein of Newcastle Disease Virus. Int J Oncol, 2004, 24: 623-634

11 Connolly S A, Leser G P, Jardetzky T S, et al. Bimolecular complementation of paramyxovirus fusion and hemagglutinin-neuraminidase proteins enhances fusion: implications for the mechanism of fusion triggering. J Virol, 2009, 83: 10857-10868

12 Dortmans J C F M, Koch G, Rottier P J M, et al. Virulence of newcastle disease virus: What is known so far? Vet Res, 2011, 42: 122

13 Connaris H, Takimoto T, Russell R, et al. Probing the sialic acid binding site of the hemagglutinin-neuraminidase of newcastle disease virus: identification of key amino acids involved in cell binding, catalysis, and fusion. J Virol, 2002, 76: 1816-1824

14 Ito Y, Komada H, Kusagawa S, et al. Fusion regulation proteins on the cell surface: Isolation and characterization of monoclonal antibodies which enhance giant polykaryocyte formation in Newcastle disease virus-infected cell lines of human origin. J Virol, 1992, 66: 5999-6007

15 Lamb R A, Jardetzky T S. Structural basis of viral invasion: lessons from paramyxovirus F. Curr Opin Struct Biol, 2007, 17: 427-436

16 Fournier P, Schirrmacher V. Oncolytic newcastle disease virus as cutting edge between tumor and host. Biology, 2013, 2: 936-975

17 Molouki A, Peeters B. Rescue of recombinant Newcastle disease virus: current cloning strategies and RNA polymerase provision systems. Arch Virol, 2017, 162: 1-12

18 Wheelock E F, Dingle J H. Observations on the repeated administration of viruses to a patient with acute leukemia. N Engl J Med, 1964, 271: $645-651$

19 Cassel W A, Garrett R E. Newcastle disease virus as an antineoplastic agent. Cancer, 1965, 18: 863-868

20 Nelson N J. Scientific interest in Newcastle disease virus is reviving. J Natl Cancer Inst, 1999, 91: 1708-1710

21 Isaacs A, Lindenmann J. Virus interference. I. The interferon. J Interferon Res, 1987, 7: 429-438

22 Lindemann J. Viruses as immunological adjuvants in cancer. Biochim Biophys Acta, 1974, 355: 49-75

23 Cassel W A, Garrett R E. Tumor immunity after viral oncolysis. J Bacteriol, 1966, 92: 792

24 Murray D R, Cassel W A, Torbin A H, et al. Viral oncolysate in the management of malignant melanoma. II. Clinical studies. Cancer, 1977, 40: $680-686$ 
25 Cassel W A, Murray D R. A ten-year follow-up on stage II malignant melanoma patients treated postsurgically with Newcastle disease virus oncolysate. Med Oncol Tumor Pharmacother, 1992, 9: 169-171

26 Batliwalla F M, Bateman B A, Serrano D, et al. A 15-year follow-up of AJCC stage III malignant melanoma patients treated postsurgically with newcastle disease virus (NDV) oncolysate and determination of alterations in the CD8 T cell repertoire. Mol Med, 1998, 4: 783-794

27 Kobayashi H. Viral xenogenisation of intact tumor cells. Adv Cancer Res, 1979, 30: 279-299

28 Lorence R M, Rood P A, Kelley K W. Newcastle Disease Virus as an antineoplastic agent: induction of tumor necrosis factor-alpha and augmentation of its cytotoxicity. J Natl Cancer Inst, 1988, 80: 1305-1312

29 Reichard K W, Lorence R M, Cascino C J, et al. Newcastle disease virus selectively kills human tumor cells. J Surg Res, 1992, 52: 448-453

30 Phuangsab A, Lorence R M, Reichard K W, et al. Newcastle disease virus therapy of human tumor xenografts: antitumor effects of local or systemic administration. Cancer Lett, 2001, 172: 27-36

31 Pecora A L, Rizvi N, Cohen G I, et al. Phase I trial of intravenous administration of PV701, an oncolytic virus, in patients with advanced solid cancers. J Clin Oncol, 2002, 20: 2251-2266

32 Lorence R M, Pecora A L, Major P P, et al. Overview of phase I studies of intravenous administration of PV701, an oncolytic virus. Curr Opin Mol Ther, 2003, 5: 618-624

33 Heicappell R, Schirrmacher V, von Hoegen P, et al. Prevention of metastatic spread by postoperative immunotherapy with virally modified autologous tumor cells. I. Parameters for optimal therapeutic effects. Int J Cancer, 1986, 37: 569-577

34 Schirrmacher V, Heicappell R. Prevention of metastatic spread by postoperative immunotherapy with virally modified autologous tumor cells. II. Establishment of specific systemic anti-tumor immunity. Clin Exp Metast, 1987, 5: 147-156

35 Von Hoegen P, Weber E, Schirrmacher V. Modification of tumor cells by a low dose of Newcastle Disease Virus. Eur J Immunol, 1988, 18: $1159-1166$

36 Schild H, von Hoegen P, Schirrmacher V. Modification of tumor cells by a low dose of Newcastle disease virus. Cancer Immunol Immunother, 1989, 28: 22-28

37 von Hoegen P, Zawatzky R, Schirrmacher V. Modification of tumor cells by a low dose of Newcastle disease virus. Cell Immunol, 1990, 126: $80-90$

38 Schirrmacher V, Haas C, Bonifer R, et al. Human tumor cell modification by virus infection: an efficient and safe way to produce cancer vaccine with pleiotropic immune stimulatory properties when using Newcastle disease virus. Gene Ther, 1999, 6: 63-73

39 Bohle W, Schlag P, Liebrich W, et al. Postoperative active specific immunization in colorectal cancer patients with virus-modified autologous tumor-cell vaccine. First clinical results with tumor-cell vaccines modified with live but avirulent newcastle disease virus. Cancer, 1990, 66: $1517-1523$

40 Ahlert T, Sauerbrei W, Bastert G, et al. Tumor-cell number and viability as quality and efficacy parameters of autologous virus-modified cancer vaccines in patients with breast or ovarian cancer. J Clin Oncol, 1997, 15: 2763

41 Ockert D, Schirrmacher V, Beck N, et al. Newcastle disease virus-infected intact autologous tumor cell vaccine for adjuvant active specific immunotherapy of resected colorectal carcinoma. Clin Cancer Res, 1996, 2: 21-28

42 Pomer S, Schirrmacher V, Thiele R, et al. Tumor response and 4 year survival-data of patients with advanced renal-cell carcinoma treated with autologous tumor vaccine and subcutaneous r-IL-2 and IFN-alpha(2b). Int J Oncol, 1995, 6: 947-954

43 Csatary L K, Eckhardt S, Bukosza I, et al. Attenuated veterinary virus vaccine for the treatment of cancer. Cancer Detect Prev, 1993, 17: 619-627

44 Feng H, Wei D, Nan G, et al. Construction of a minigenome rescue system for Newcastle disease virus strain Italien. Arch Virol, 2011, 156: 611616

45 Wei D, Sun N, Nan G, et al. Construction of recombinant Newcastle disease virus Italien strain for oncolytic virotherapy of tumors. Hum Gene Ther, 2012, 23: 700-710

46 Ahlert T, Schirrmacher V. Isolation of a human melanoma adapted Newcastle disease virus mutant with highly selective replication patterns. Cancer Res, 1990, 50: 5962-5968

47 Karcher J, Dyckhoff G, Beckhove P, et al. Antitumor vaccination in patients with head and neck squamous cell carcinomas with autologous virusmodified tumor cells. Cancer Res, 2004, 64: 8057-8061

48 Steiner $\mathrm{H} \mathrm{H}$, Bonsanto $\mathrm{M} \mathrm{M}$, Beckhove $\mathrm{P}$, et al. Antitumor vaccination of patients with glioblastoma multiforme: a pilot study to assess feasibility, safety, and clinical benefit. J Clin Oncol, 2004, 22: 4272-4281 
49 Schulze T, Kemmner W, Weitz J, et al. Efficiency of adjuvant active specific immunization with Newcastle disease virus modified tumor cells in colorectal cancer patients following resection of liver metastases: results of a prospective randomized trial. Cancer Immunol Immunother, 2009, 58: 61-69

50 Liang W, Wang H, Sun T M, et al. Application of autologous tumor cell vaccine and NDV vaccine in treatment of tumors of digestive tract. World J Gastroenterol, 2003, 9: 495-498

51 Bian $\mathrm{H}$, Wilden $\mathrm{H}$, Fournier P, et al. In vivo efficacy of systemic tumor targeting of a viral RNA vector with oncolytic properties using a bispecific adapter protein. Int J Oncol, 2006, 29: 1359-1369

$52 \mathrm{Li} \mathrm{Y} \mathrm{L,} \mathrm{Wu} \mathrm{J,} \mathrm{Wei} \mathrm{D,} \mathrm{et} \mathrm{al.} \mathrm{Newcastle} \mathrm{disease} \mathrm{virus} \mathrm{represses} \mathrm{the} \mathrm{activation} \mathrm{of} \mathrm{human} \mathrm{hepatic} \mathrm{stellate} \mathrm{cells} \mathrm{and} \mathrm{reverses} \mathrm{the} \mathrm{development} \mathrm{of}$ hepatic fibrosis in mice. Liver Int, 2009, 29: 593-602

53 Bai L, Koopmann J, Fiola C, et al. Dendritic cells pulsed with viral oncolysates potently stimulate autologous T cells from cancer patients. Int J Oncol, 2002, 21: 685-694

54 Elankumaran S, Rockemann D, Samal S K. Newcastle disease virus exerts oncolysis by both intrinsic and extrinsic caspase-dependent pathways of cell death. J Virol, 2006, 80: 7522-7534

55 Schirrmacher V, Fournier P, Schlag P. Autologous tumor cell vaccines for post-operative active-specific immunotherapy of colorectal carcinoma: long-term patient survival and mechanism of function. Expert Rev Vaccines, 2014, 13: 117-130

56 Fournier P, Schirrmacher V. Bispecific antibodies and trispecific immunocytokines for targeting the immune system against cancer. BioDrugs, 2013, 27: $35-53$

57 Schirrmacher V, Bihari A S, Stücker W, et al. Long-term remission of prostate cancer with extensive bone metastases upon immuno- and virotherapy: a case report. Oncol Lett, 2014, 8: 2403-2406

58 Ni J, Schirrmacher V, Fournier P. The hemagglutinin-neuraminidase gene of Newcastle Disease Virus: a powerful molecular adjuvant for DNA anti-tumor vaccination. Vaccine, 2010, 28: 6891-6900

59 Koks C A, Garg A D, Ehrhardt M, et al. Newcastle disease virotherapy induces long-term survival and tumor-specific immune memory in orthotopic glioma through the induction of immunogenic cell death. Int J Cancer, 2015, 136: E313-E325

60 Fournier P, Wilden H, Schirrmacher V. Importance of retinoic acid-inducible gene I and of receptor for type I interferon for cellular resistance to infection by Newcastle disease virus. Int J Oncol, 2011, doi: 10.3892/ijo.2011.1222

61 Zaslavsky E, Hershberg U, Seto J, et al. Antiviral response dictated by choreographed cascade of transcription factors. J Immunol, 2010, 184: 2908-2917

62 Schirrmacher V, Schlude C, Weitz J, et al. Strong T-cell costimulation can reactivate tumor antigen-specific T cells in late-stage metastasized colorectal carcinoma patients: Results from a phase I clinical study. Int J Oncol, 2015, 46: 71-77

63 Schirrmacher V, Stücker W, Lulei M, et al. Long-term survival of a breast cancer patient with extensive liver metastases upon immune and virotherapy: a case report. Immunotherapy, 2015, 7: 855-860

64 Wei D, Li Q, Wang X L, et al. Oncolytic Newcastle disease virus expressing chimeric antibody enhanced anti-tumor efficacy in orthotopic hepatoma-bearing mice. J Exp Clin Cancer Res, 2015, 34: 153

65 Mansour M, Palese P, Zamarin D. Oncolytic specificity of Newcastle Disease Virus is mediated by selectivity for apoptosis-resistant cells. J Virol, 2011, 85: 6015-6023

66 Zamarin D, Holmgaard R B, Subudhi S K, et al. Localized oncolytic virotherapy overcomes systemic tumor resistance to immune checkpoint blockade immunotherapy. Sci Transl Med, 2014, 6: 226-332

67 An Y, Liu T, He J, et al. Recombinant Newcastle disease virus expressing P53 demonstrates promising antitumor efficiency in hepatoma model. J Biomed Sci, 2016, 23: 55

68 Abdullah J M, Mustafa Z, Ideris A. Newcastle disease virus interaction in targeted therapy against proliferation and invasion pathways of glioblastoma multiforme. Biomed Res Int, 2014, 2014: 1-11

69 Al-Shammari A M, Rameez H, Al-Taee M F. Newcastle disease virus, rituximab, and doxorubicin combination as anti-hematological malignancy therapy. Oncolytic Virother, 2016, 5: 27-34

70 Schirrmacher V. Fifty years of clinical application of newcastle disease virus: time to celebrate! Biomedicines, 2016, 4: 16

71 Ricca J M, Oseledchyk A, Walther T, et al. Pre-existing immunity to oncolytic virus potentiates its immunotherapeutic efficacy. Mol Ther, 2018, 26: $1008-1019$

72 Chaurasiya S, Chen N G, Fong Y. Oncolytic viruses and immunity. Curr Opin Immunol, 2018, 51: 83-90 
73 McGinnes L W, Morrison T G. Inhibition of receptor binding stabilizes Newcastle disease virus HN and F protein-containing complexes. J Virol, 2006, 80: 2894-2903

74 Schirrmacher V, Fournier P. Newcastle diasease virus: a promising vector for viral therapy, immune therapy, and gene therapy of cancer. Methods Mol Biol 2009, 542: 565-605

75 Fiola C, Peeters B, Fournier P, et al. Tumor selective replication of Newcastle disease virus: association with defects of tumor cells in antiviral defence. Int J Cancer, 2006, 119: 328-338

76 Wilden H, Fournier P, Zawatzky R, et al. Expression of RIG-I, IRF3, IFN- $\beta$ and IRF7 determines resistance or susceptibility of cells to infection by Newcastle disease virus. Int J Oncol, 2009, 34: 971-982

77 Elankumaran S, Chavan V, Qiao D, et al. Type I interferon-sensitive recombinant Newcastle disease virus for oncolytic virotherapy. J Virol, 2010, 84: $3835-3844$

78 Puhlmann J, Puehler F, Mumberg D, et al. Rac1 is required for oncolytic NDV replication in human cancer cells and establishes a link between tumorigenesis and sensitivity to oncolytic virus. Oncogene, 2010, 29: 2205-2216

79 Jarahian M, Watzl C, Fournier P, et al. Activation of natural killer cells by Newcastle disease virus hemagglutinin-neuraminidase. J Virol, 2009, 83: $8108-8121$

80 Washburn B, Weigand M A, Grosse-Wilde A, et al. TNF-related apoptosis-inducing ligand mediates tumoricidal activity of human monocytes stimulated by newcastle disease virus. J Immunol, 2003, 170: 1814-1821

81 Schirrmacher V, Bai L, Umansky V, et al. Newcastle disease virus activates macrophages for anti-tumor activity. Int J Oncol, 2000, 16: 363-373

82 Umansky V, Shatrov V A, Lehmann V, et al. Induction of NO synthesis in macrophages by Newcastle disease virus is associated with activation of nuclear factor- $\mathrm{\kappa B}$. Int Immunol, 1996, 8: 491-498

83 Chlichlia K, Peter M E, Rocha M, et al. Caspase activation is required for nitric oxide-mediated, CD95(APO-1/Fas)-dependent and independent apoptosis in human neoplastic lymphoid cells. Blood, 1998, 91: 4311-4320

84 Zhao L, Niu C, Shi X, et al. Dendritic cells loaded with the lysate of tumor cells infected with Newcastle Disease Virus trigger potent anti-tumor immunity by promoting the secretion of IFN- $\gamma$ and IL-2 from T cells. Oncol Lett, 2018, 16: 1180-1188

85 Song H, Zhong L P, He J, et al. Application of Newcastle disease virus in the treatment of colorectal cancer. World J Clin Cases, 2019, 7: 21432154

86 Liao Y, Wang H X, Mao X, et al. RIP1 is a central signaling protein in regulation of TNF- $\alpha /$ TRAIL mediated apoptosis and necroptosis during Newcastle disease virus infection. Oncotarget, 2017, 8: 43201-43217

87 Molouki A, Hsu Y T, Jahanshiri F, et al. The matrix (M) protein of Newcastle disease virus binds to human bax through its BH3 domain. Virol J, 2011, 8: 385

88 Altieri D C. Survivin — the inconvenient IAP. Semin Cell Dev Biol, 2015, 39: 91-96

89 Shao X, Wang X, Guo X, et al. STAT3 contributes to oncolytic newcastle disease virus-induced immunogenic cell death in melanoma cells. Front Oncol, 2019, 9: 436

90 Wang X, Shao X, Gu L, et al. Targeting STAT3 enhances NDV-induced immunogenic cell death in prostate cancer cells. J Cell Mol Med, 2020, 24: 4286-4297

91 Li Y, Wang L X, Yang G, et al. Efficient cross-presentation depends on autophagy in tumor cells. Cancer Res, 2008, 68: 6889-6895

92 Bian J, Wang K, Kong X, et al. Caspase- and p38-MAPK-dependent induction of apoptosis in A549 lung cancer cells by Newcastle disease virus. Arch Virol, 2011, 156: 1335-1344

93 Ye T, Jiang K, Wei L, et al. Oncolytic Newcastle disease virus induces autophagy-dependent immunogenic cell death in lung cancer cells. Am J Cancer Res, 2018, 8: 1514-1527

94 Jiang K, Li Y, Zhu Q, et al. Pharmacological modulation of autophagy enhances Newcastle disease virus-mediated oncolysis in drug-resistant lung cancer cells. BMC Cancer, 2014, 14: 551

95 Kan X, Yin Y, Song C, et al. Newcastle-disease-virus-induced ferroptosis through nutrient deprivation and ferritinophagy in tumor cells. iScience, 2021, 24: 102837

96 Schirrmacher V, van Gool S, Stuecker W. Breaking therapy resistance: an update on oncolytic newcastle disease virus for improvements of cancer therapy. Biomedicines, 2019, 7:66

97 Burman B, Pesci G, Zamarin D. Newcastle disease virus at the forefront of cancer immunotherapy. Cancers, 2020, 12: 3552 


\title{
Oncolytic Newcastle disease virus: a new opportunity in the era of tumor immunotherapy
}

\author{
DU Sha ${ }^{1}$, JIANG $\mathrm{Ke}^{2}$, DING Chan ${ }^{3}$, XU Qing ${ }^{4}$, LI WenBin ${ }^{5} \&$ MENG SongShu ${ }^{1}$ \\ 1 Institute of Cancer Stem Cell, Dalian Medical University, Dalian 116044, China; \\ 2 Center for Single-Cell Omics and Disease Research, Shanghai Jiao Tong University School of Medicine, Shanghai 200025, China; \\ 3 Department of Avian Infectious Diseases, Shanghai Veterinary Research Institute, Chinese Academy of Agricultural Sciences, \\ Shanghai 200241, China; \\ 4 Department of Medical Oncology, Shanghai Tenth People's Hospital, Tongji University, Shanghai 200072, China; \\ 5 Department of Neuro-oncology, Neurosurgery center, Beijing Tiantan Hospital, Capital Medical University, Beijing 100070, China
}

Oncolytic virus therapy is one of the hot topics in tumor immunotherapy. As a novel anticancer agent, oncolytic Newcastle disease virus (NDV) uses antiviral defense defects in the tumor cells for tumor treatment. NDV selectively infects, replicates and cracks tumor cells, enhances tumor antigen exposure, promotes the recruitment of immune cells, and activates antitumor immune response, thus playing a role in antitumor immunity. Based on the unique antitumor properties of oncolytic NDV and its interaction with patients' immune systems, the combination of oncolytic NDV and other immunotherapies can enhance the antitumor efficacy and have broad applications. This paper reviews the structure and mechanism of oncolytic NDV, preclinical research and clinical application of oncolytic NDV in tumor therapy, and prospects of combined immunotherapy.

oncolytic virus, Newcastle disease virus, tumor immunotherapy, combined immunotherapy

doi: $10.1360 /$ SSV-2021-0434 